PROCEEDINGS OF THE

AMERICAN MATHEMATICAL SOCIETY

Volume 127, Number 11, Pages 3305-3308

S 0002-9939(99)05107-2

Article electronically published on May 13, 1999

\title{
SUM OF CANTOR SETS: SELF-SIMILARITY AND MEASURE
}

\author{
PEDRO MENDES
}

(Communicated by Michael Handel)

\begin{abstract}
In this note it is shown that the sum of two homogeneous Cantor sets is often a uniformly contracting self-similar set and it is given a sufficient condition for such a set to be of Lebesgue measure zero (in fact, of Hausdorff dimension less than one and positive Hausdorff measure at this dimension).
\end{abstract}

\section{Definitions And Results}

The study of the arithmetic difference (sum) of two Cantor sets has been of great interest for the homoclinic bifurcations of dynamical systems, since the last decade. For a detailed presentation of this subject we refer the reader to [7]. About 1987, J. Palis made the following

Conjecture. If the sum of two affine Cantor sets has positive Lebesgue measure, then it contains an interval.

Many articles have been written on this conjecture; see [4], [5], [6] and [8].

Another subject which has been of great interest since the work of B. Mandelbrot ([3]) is the study of fractal dimensions, introduced by Hausdorff in the twenties. The most useful source of examples in this context is given by self-similar sets. Several efforts have been made ([1], [2], [9]) to solve the following

Question. Is it true that if a self-similar set has positive Lebesgue measure, then it contains an interval?

Our first result (Theorem A below) gives an indication that the conjecture and the question above are related to each other. We are going to state it precisely.

Let $f_{i}: x \in \mathbb{R} \mapsto c_{i} x+a_{i} \in \mathbb{R}, 0<c_{i}<1, i=1, \ldots, r$, be affine contractions of the real line. There is a unique compact and nonempty set $A \subset \mathbb{R}$ such that $A=f_{1}(A) \cup \cdots \cup f_{r}(A)([2])$. This set is called a self-similar set, and it is called a uniformly contracting self-similar set whenever $c_{1}=\cdots=c_{r}=c$.

Let $\mathcal{H}(2 r+1)$ be the set of all vectors $\vec{\lambda}=\left(\lambda, \lambda_{1}^{\prime}, \lambda, \ldots, \lambda_{r}^{\prime}, \lambda\right) \in \mathbb{R}^{2 r+1}$, such that $\lambda>0, \lambda_{i}^{\prime}>0, i=1, \ldots, r$; and $\lambda+\lambda_{1}^{\prime}+\lambda+\cdots+\lambda_{r}^{\prime}+\lambda=1$. Let $C(\vec{\lambda})$ be the self-similar set obtained, as above, using the contractions $f_{i}(x)=\lambda x+a_{i}$, with $a_{0}=0, a_{i}=\lambda+\lambda_{1}^{\prime}+\lambda+\cdots+\lambda_{i}^{\prime}, i=1, \ldots, r$. The set $C(\vec{\lambda})$ is called a homogeneous Cantor set. We have the following

Received by the editors February 6, 1998.

1991 Mathematics Subject Classification. Primary 28A78, 58F14.

Key words and phrases. Self-similar set, homogeneous Cantor set, Hausdorff dimension, Hausdorff measure.

(c)1999 American Mathematical Society 
Theorem A. There is a dense set $\mathcal{D} \subset \mathcal{H}(2 r+1) \times \mathcal{H}(2 s+1)$, such that if $(\vec{\lambda}, \vec{\gamma}) \in$ $\mathcal{D}$, then $C(\vec{\lambda})+C(\vec{\gamma})=\{x+y: x \in C(\vec{\lambda}), y \in C(\vec{\gamma})\}$ is a uniformly contracting self-similar set.

We also obtain a sufficient condition for a uniformly contracting self-similar set to be of Lebesgue measure zero. Let $A$ be a uniformly contracting self-similar set, as above. We may assume, without loss of generality (see next section for explanation), that $0=a_{1}<a_{2}<\cdots<a_{r}=1-c<1$, where $c$ is the uniform contracting ratio. We consider the set $\mathcal{A}$ of analytic functions $f$ given by $f(x)=d_{0}+d_{1} x+d_{2} x^{2}+\ldots$, where $d_{k}=a_{i}-a_{j}$, for some $i, j=1, \ldots, r$, for all $k=0,1,2, \ldots$, and $d_{0} \neq 0$. Our second result is the following

Theorem B. If $f(c) \neq 0$ for all $f \in \mathcal{A}$, then the uniformly contracting self-similar set $A$ has Hausdorff dimension less than one and positive Hausdorff measure at this dimension. In particular, A has Lebesgue measure zero.

Finally, we believe that Theorem A is true in the more general context of all affine Cantor sets.

\section{Preliminaries}

Let $g_{i}: \mathbb{R} \rightarrow \mathbb{R}$ be given by $g_{i}(x)=c x+b_{i}, i=1, \ldots, r$, with $b_{1}<\cdots<b_{r}$. Consider the affine bijection $S: \mathbb{R} \rightarrow \mathbb{R}$ given by $S(x)=\frac{b_{r}-b_{1}}{1-c} x+\frac{b_{1}}{1-c}$. It is easy to verify that $f_{i}=S^{-1} \circ g_{i} \circ S$ is given by $f_{i}(x)=c x+a_{i}$, with $a_{i}=(1-c) \frac{b_{i}-b_{1}}{b_{r}-b_{1}}$. Then, $0=a_{1}<a_{2}<\cdots<a_{r}=1-c$. The existence and uniqueness of nonempty compact sets $B$ and $A$ such that $B=g_{1}(B) \cup \cdots \cup g_{r}(B)$ and $A=f_{1}(A) \cup \cdots \cup f_{r}(A)$, was proved in ([2]). By our construction, it is clear that $S(A)=B$. Thus, we can work with $A$ instead of $B$, without loss of generality.

Now, we are going to describe a useful construction of $A$. Let $A_{0}=[0,1]$. It is easy to see that $\left(f_{i_{n}} \circ \cdots \circ f_{i_{1}}\right)\left(A_{0}\right)$ is an interval of length $c^{n}$. The union of all such intervals is denoted by $A_{n}$ and is called $n$-step of the construction of $A$. Moreover, $\left(f_{i_{n}} \circ \cdots \circ f_{i_{1}}\right)\left(A_{0}\right)$ is called an interval of $A_{n}$. Then, we have that $A=\bigcap_{n=0}^{\infty} A_{n}$.

Using this construction, we can characterize an element $a \in A$ by the existence of a sequence $a_{i_{k}}$, with $a_{i_{k}} \in\left\{a_{1}, \ldots, a_{r}\right\}$, such that $a=\sum_{k=0}^{\infty} a_{i_{k}} c^{k}$. The reciprocal of this fact will be useful in the next section. It can be stated as follows. Let $\left\{b_{1}, \ldots, b_{r}\right\}$ be a given finite set and let $c \in(0,1)$. The set $B$ of the numbers $\sum_{k=0}^{\infty} b_{i_{k}} c^{k}$, with $b_{i_{k}} \in\left\{b_{1}, \ldots, b_{r}\right\}$, is the unique nonempty compact set satisfying the equation $B=g_{1}(B) \cup \cdots \cup g_{r}(B)$, with $g_{i}$ as above (see [2]). This fact will be used in the proof of Theorem A.

Another important tool we are going to use in the next section is the strong open set condition SOSC. We say that the self-similar set $A$ above satisfies the SOSC if there is an open set $V \supset A$, such that $f_{i}(V) \cap f_{j}(V)=\emptyset$, whenever $i \neq j$, and $f_{i}(V) \subset V$, for all $i=1, \ldots, r$. An important result of $([2])$ says that if the selfsimilar set $A$ satisfies the SOSC, then A has Hausdorff dimension less than one and positive Hausdorff measure at this dimension. We are going to use this fact in the proof of Theorem B. 


\section{Proofs of The Results}

3.1 Proof of Theorem A. Let us define the set $\mathcal{D}$. An element $(\vec{\lambda}, \vec{\gamma})$ belongs to $\mathcal{D}$ if, and only if, there are a real number $\mu \in(0,1)$ and positive integers $p$ and $q$ such that $\lambda=\mu^{p}$ and $\gamma=\mu^{q}$. Recall that $\vec{\lambda}=\left(\lambda, \lambda_{1}^{\prime}, \lambda, \ldots, \lambda_{r}^{\prime}, \lambda\right)$ and $\vec{\gamma}=\left(\gamma, \gamma_{1}^{\prime}, \gamma, \ldots, \gamma_{s}^{\prime}, \gamma\right)$ are elements of $\mathcal{H}(2 r+1)$ and $\mathcal{H}(2 s+1)$, respectively. For a fixed $\gamma \in(0,1)$ the continuous decreasing function $t \mapsto \gamma^{t}$ assume all values in the interval $(0,1)$, as $t$ varies from 0 to $\infty$. Therefore, an appropriate choice for the rational number $p / q>0$, with small perturbations of $\lambda_{i}^{\prime}, i=1, \ldots, r$, allow us to approximate an element $(\vec{\lambda}, \vec{\gamma})$ of $\mathcal{H}(2 r+1) \times \mathcal{H}(2 s+1)$ by an element of the form

$$
\left(\left(\gamma^{p / q}, \lambda_{1}^{\prime}, \gamma^{p / q}, \ldots, \lambda_{r}^{\prime}, \gamma^{p / q}\right),\left(\gamma, \gamma_{1}^{\prime}, \gamma, \ldots, \gamma_{s}^{\prime}, \gamma\right)\right) .
$$

Therefore, it is enough to choose $\mu=\gamma^{1 / q}$ to see that this element is in $\mathcal{D}$.

It remains to prove that if $(\vec{\lambda}, \vec{\gamma}) \in \mathcal{D}$, then $C(\vec{\lambda})+C(\vec{\gamma})$ is a uniformly contracting self-similar set. As we saw in the previous section, an element $x$ of $C(\vec{\lambda})$ is given by $x=\sum_{k=0}^{\infty} a_{i_{k}}\left(\mu^{p}\right)^{k}$ and an element $y$ of $C(\vec{\gamma})$ is given by $y=\sum_{k=0}^{\infty} b_{j_{k}}\left(\mu^{q}\right)^{k}$, because $(\vec{\lambda}, \vec{\gamma}) \in \mathcal{D}$. Then $x+y$ is given by a power series in $c=\mu^{p q}$, whose coefficients vary in the finite set of all sums of the form $\sum_{k=0}^{q-1} a_{i_{k}} \mu^{k p}+\sum_{k=0}^{p-1} b_{j_{k}} \mu^{k q}$. Thus, again by the previous section, it follows that $C(\vec{\lambda})+C(\vec{\gamma})$ is a uniformly contracting self-similar set, with contracting ratio $c$.

3.2 Proof of Theorem B. Let $I=[a, b] \subset(0,1)$ be a closed interval such that $c \in I$. We are going to be more precise about the set $\mathcal{A}:$ an element $f \in \mathcal{A}$ is a power series $f(x)=\sum_{k=0}^{\infty} d_{k} x^{k}$, with $x \in I$ and $d_{k} \in D$, for all $k$. Here $D \subset(-1,1)$ is the finite set $\left\{a_{i}-a_{j}, i, j=1, \ldots, r\right\}$, which is symmetric with respect to $0 \in D$. Moreover, if $f \in \mathcal{A}$, then $d_{0} \neq 0$.

We are going to prove that $\mathcal{A}$ is compact in the uniform topology. In fact, let $\Lambda$ be the set of sequences $d=\left(d_{0}, d_{1}, d_{2}, \ldots\right) \in(D \backslash\{0\}) \times D \times D \times \ldots$ with the product topology. Let $\Phi: \Lambda \rightarrow \mathcal{A}$ be defined by $[\Phi(d)](x)=\sum_{k=0}^{\infty} d_{k} x^{k}$. By the definition of $\mathcal{A}$, it follows that $\Phi$ is onto. If $d, d^{\prime} \in \Lambda$ are sufficiently near, then the distance between $\Phi(d)$ and $\Phi\left(d^{\prime}\right)$ in the uniform topology is bounded by $b^{n} \frac{2(1-c)}{1-b}$, where $n$ is arbitrarily big. This implies that $\Phi$ is continuous and therefore $\mathcal{A}$ is compact, as we claimed above.

The hypothesis that $f(c) \neq 0$, for all $f \in \mathcal{A}$, assures the existence of a number $\rho>0$ such that $|f(c)| \geq \rho$, for all $f \in \mathcal{A}$, because $\mathcal{A}$ is compact. Now we choose $n_{0}>0$ such that $\rho>c^{n}$, whenever $n \geq n_{0}$. Consider the $n$-step $A_{n}$ of the construction of $A$, for some $n \geq n_{0}$, as described in the previous section. We are going to show that $f_{i}\left(A_{n}\right) \cap f_{j}\left(A_{n}\right)=\emptyset$, for all $i, j=1, \ldots, r, i \neq j$. In fact, suppose by contradiction that $f_{i}\left(A_{n}\right) \cap f_{j}\left(A_{n}\right) \neq \emptyset$. This means that there are intervals $I, J$ of $A_{n}$ such that $f_{i}(I) \cap f_{j}(J) \neq \emptyset$, that is, the difference between the left ends of the intervals $f_{i}(I)$ and $f_{j}(J)$ is bounded in modulus by $c^{n+1}$. This difference is given by 
$\sum_{k=0}^{n+1} d_{k} c^{k}$, where $d_{0}=a_{i}-a_{j} \neq 0$. Thus, the function $f(x)=\sum_{k=0}^{n+1} d_{k} x^{k}$ is an element of $\mathcal{A}$ and then $|f(c)| \geq \rho>c^{n+1}$, which is a contradiction.

Let $V_{i}$ be the $c \varepsilon$-neighbourhood of $f_{i}\left(A_{n}\right), i=1, \ldots, r$. The number $\varepsilon>0$ can be chosen such that the open sets $V_{1}, \ldots, V_{r}$ are pairwise disjoint, because the sets $f_{1}\left(A_{n}\right), \ldots, f_{r}\left(A_{n}\right)$ are compact and pairwise disjoint. Let $V$ be the $\varepsilon$-neighbourhood of $A_{n}$. It is clear that $f_{i}(V)=V_{i}$, for all $i$. Moreover, $V \supset V_{i}$ because $A_{n} \supset f_{i}\left(A_{n}\right)$. Thus, the self-similar set $A$ satisfies the strong open set condition and therefore has Hausdorff dimension less than one and positive Hausdorff measure at this dimension.

\section{REFERENCES}

1. C. Brant, S. Graf, Self-similar sets 7. A characterization of self-similar fractals with positive Hausdorff measure, Proc. Amer. Math. Soc. 114 (1992), 995 - 1001. MR 93d:28014

2. J. E. Hutchinson, Fractals and self-similarity, Indiana Univ. Math. J. 30 (1981), $713-747$. MR 82h: 49026

3. B. Mandelbrot,, Fractals, Form, Chance and Dimension, Freeman, San Francisco, 1977. MR $\mathbf{5 7 : 1 1 2 2 4}$

4. P. Mendes, F. Oliveira, On the topological structure of the arithmetic sum of two Cantor sets., Nonlinearity $\mathbf{7}$ (1994), 329 - 343. MR 95j:58123

5. C. G. T. de A. Moreira, Stable intersections of Cantor sets and homoclinic bifurcations, Ann. Inst. Henri Poincaré: Analyse non linéaire 13 (1996), 741 - 781.

6. C. G. T. de A. Moreira, C. Yoccoz, Stable intersections of Cantor sets with large Hausdorff dimension (to appear).

7. J. Palis, F. Takens, Hyperbolicity and sensitive chaotic dynamics at homoclinic bifurcations: fractal dimensions and infinitely many attractors., Cambridge Univ. Press, 1993. MR 94h:58129

8. B. Solomyak, On the measure of arithmetic sums of Cantor sets., Indag. Mathem., N.S. 8 (1997), 133 - 141. CMP 98:12

9. M. P, W. Zerner, Weak separation properties for self-similar sets, Proc. Amer. Math. Soc. 124 (1996), 3529 - 3539. MR 97c:54035

Departamento de Matemática, ICEx, ufmg Av. Antonio Carlos 662731270.901 Belo Horizonte, MG, Brazil

E-mail address: pmendes@mat.ufmg.br 